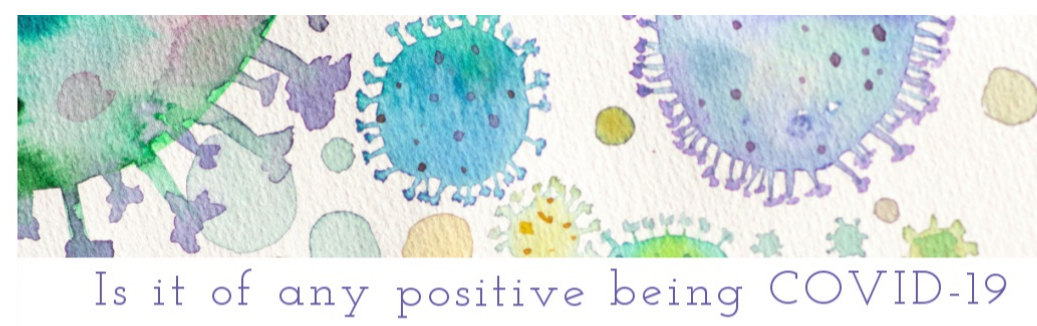

positive?

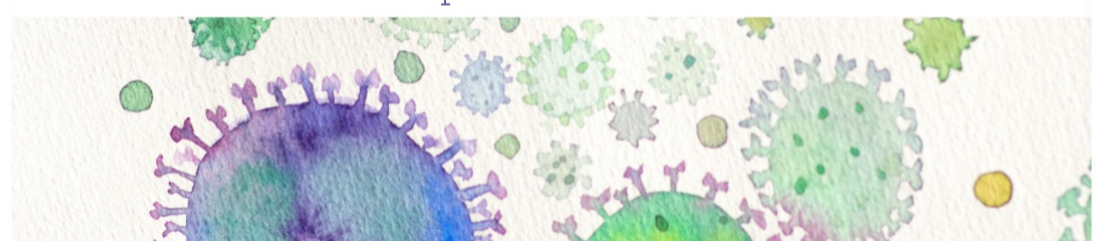

\title{
Is it of any positive being COVID-19 positive?: Cross- protection hypothesis
}

\author{
Muath Alser ${ }^{1}$ \\ 1 Faculty of Medicine, Cairo University
}

Funding: The author(s) received no specific funding for this work.

Potential competing interests: The author(s) declared that no potential competing interests exist.

\section{Abstract}

Many pathogens have been reported to induce cross-protective immune responses against other related and unrelated pathogens due to shared epitopes or induction of trained immunity.

Herein, I review the evidence we have so far on the possible SARS-CoV-2 cross-reactions with other pathogens, and the immune modulatory effects it could induce, which could lead to beneficial effects against other diseases among COVID-19-recovered immunocompetent individuals.

\section{Introduction}

At the time of this writing, around 167 million people were tested positive for COVID-19 and more than 3.47 million died worldwide.(1) For sure, this is the tip of a hidden iceberg due to limited testing capacity and difficulty to detect asymptomatic infection.(2)

Looking at the scientific literature on this pandemic, most of characterization studies focus on the negative effects of this pandemic on the human health and other life aspects. Reported symptoms were not limited to respiratory or gastrointestinal symptoms but included most of human body; literally from head (e.g. 
headache and foggy brain) to toe (COVID-19 toes)! $(3,4)$ Moreover, a growing body of literature coined the term post-COVID-19 syndrome or long COVID-19 to describe unexplained long lasting symptoms after being virus-free.(5)

On the other hand, few studies timidly addressed some positive effects of COVID-19, most of them are indirectly linked to the virus e.g. effect of mitigation measures on air pollution,(6) or wearing facemasks on spread of influenza, or hand hygiene on spread of enteroviruses.(7)

But the question is: Is there any direct positive impact of SARS-CoV-2 -the virus itself- on individuals' health during the infection period or afterward?

\section{The hypothesis}

SARS-CoV-2 could induce protective immune responses against its different variants, other human coronaviruses (HCoVs) (e.g. SARS, MERS, common cold coronaviruses), other non-coronaviruses (e.g. VZV, EBV, Herpesvirus, Hepatitis and Influenza), and even other pathogens including bacteria, fungi, or parasites. In addition, it could cause more tolerance to self-antigens and modulate immune response to cancer cells and autoimmune diseases. These responses could be short-term limited to the active infection period, or long lasting for months to years after the infection.

\section{Human Immunity and cross-protection}

The infection net result is generally determined by two main factors: the pathogen virulence and the host immune response.

Our human immune system is of two types: innate and acquired. Cells of innate immunity (natural killers (NK) and granulocytes) recognise pathogen and its associated damage through pattern recognition receptors (PRRs), which in turn activate a conserved defence pathways specific to this pathogen category. The way our innate immunity classify invading pathogens differs from microbiologists' taxonomy, as it depends on the molecular patterns essential to pathogen survival -to be targeted-, and the patterns of the tissue damage induced. These patterns could be shared with wide variety of pathogens and diseases.(8)

On the other immunity arm, adaptive immune cells (dendritic cells (DC) and T\&B lymphocytes) do not respond to the invading pathogen as whole, but to specific parts of it called epitopes inducing a stronger, long lasting, and more specific responses.

Epitope is any part of the antigen that makes contact with part of the antibody called paratope, or bind with T-cell receptor after being presented on antigen presenting cell (APC). So antibodies recognise circulating pathogens through the outermost exposed epitopes, while T-cells recognise shorter peptides 
from any part of the pathogen after cellular processing and presenting by APC.(9)

For that, an epitope could be a set of continuous subunits (e.g. amino acids), or discontinuous subunits that come close together in the spatial conformation. This leads to wide range of combinations of epitopes of the same antigen depending on its chemical and physical state. Epitopes and paratopes complementary-binding depends on shape and charge more than subunits sequence. So an epitope could bind to unrelated paratope and vice versa.(10)

Based on this, a protective immunity from wide range of pathogens could be achieved due to exposure to other unrelated pathogens due to shared immunogenic epitopes, shared innate immunity pathogen patterns, or shared tissue damage patterns.

\section{How long does cross-protection last?}

It has been a fact for decades that adaptive immunity has long-term (months-decades) memory, achieved through gene recombination and clonal selection of B and T cells within germinal centres of the secondary lymphoid tissues, which -these cells- in turn rapidly reactivate once exposed to their complementary epitopes.

Interestingly, during the last decade, a growing body of literature has suggested the term "trained immunity" to describe the newly observed memory properties of the innate immune cells, which mediated by epigenetic and metabolic reprogramming.(11) However, trained immunity is thought to be reversible and short lived (could last for months up to 5 years)(12)

So cross-reactive innate and adaptive immune cells with memory properties could lead to cross protection from other related and unrelated pathogens during the infection period, shortly after it, or even for yearslong.

\section{Examples of non-specific cross-reactions}

In mice studies, cross reactivity has been reported among viruses like flaviviruses ((Dengue virus (DV), yellow fever virus (YFV) and Japanese encephalitis virus (JEV)),(13) between viruses and bacteria as in herpesvirus cross-reaction with Listeria monocytogenes and Yersinia pestis,(14) and also between fungi and bacteria as in Candida albicans cross reaction with Acinetobacter baumannii.(15)

In human studies, cross-reactivity has been reported between related viruses like Hepatitis C virus (HCV) with Hepatitis A, B, and D viruses.(16) Interestingly, HCV also cross-reacted with unrelated viruses like Influenza A virus,(17)and Adenovirus.(18) Similarly, Epstein-Barr Virus (EBV) cross-reacted with the unrelated Influenza virus.(19) 
Vaccines like BCG(Bacillus Calmette Guerin), OPV(Oral Polio Vaccine), DPT(Diphtheria, Pertussis, Tetanus), smallpox and measles have shown non-specific protection against other pathogens and diseases.(20)

For example, BCG -a live attenuated Mycobacterium Bovis vaccine introduced more than 90 years ago to protect primarily from tuberculosis-has shown wide beneficial effects against viruses like: Yellow fever,(21) HPV(Human papilloma virus),(22) RSV(Respiratory Syncytial Virus),(23) HSV(Herpes Simplex Virus), (24)parasitic diseases as malaria,(25) fungi as candida albicans, and bacteria like staphylococci.(26)

Moreover, BCG vaccine has been evident to induce an anticancer immune response against bladder cancer,(27) leukaemia,(28) lymphoma,(29) and melanoma.(30) More surprisingly, beneficial effects of BCG vaccine has been reported against chronic diseases like bronchial asthma,(31) and autoimmune diseases like: T1D (type-1 Diabetes) and MS (Multiple Sclerosis).(32, 33)

The list is so long but all refer to the same concept of immune modulation through induction of epitopespecific adaptive cross-protection, trained innate immunity, or both.

\section{Evidence in the context of COVID-19}

\section{Cross-variant immunity}

Majority of COVID-19 patients develop SARS-CoV-2 specific neutralizing antibodies along with variable levels of $\mathrm{CD}^{+}{ }^{+}$and $\mathrm{CD}^{+}{ }^{+} \mathrm{T}$ cell responses.(34, 35). Substantial immune memory against SARS-CoV-2 has been reported among $95 \%$ of recovered individuals after 6-8 months.(36)

These durable immune responses are capable to cross-protect from different variants of SARS-CoV-2, which could be further boosted by single dose of COVID-19 mRNA vaccine.(37) This cross-variant immunity could explain the scarceness of COVID-19 confirmed-reinfection cases worldwide (70 cases so far).(38)

\section{Pan-coronaviral immunity}

There are six human coronaviruses (HCoVs) that share same family with SARS-CoV-2: SARS-CoV, MERSCoV, and the four endemic human coronaviruses (HCoV-OC43, -HKU1, -NL63, and -229E) (figure 1). Up to this point, there is no full epitope mapping for SARS-CoV-2, however, hundreds of priori immunogenic epitopes of viral S, $\mathrm{M}$ and $\mathrm{N}$ regions have been predicted based on mapping of the structurally most similar virus; SARS-CoV.(39, 40) 


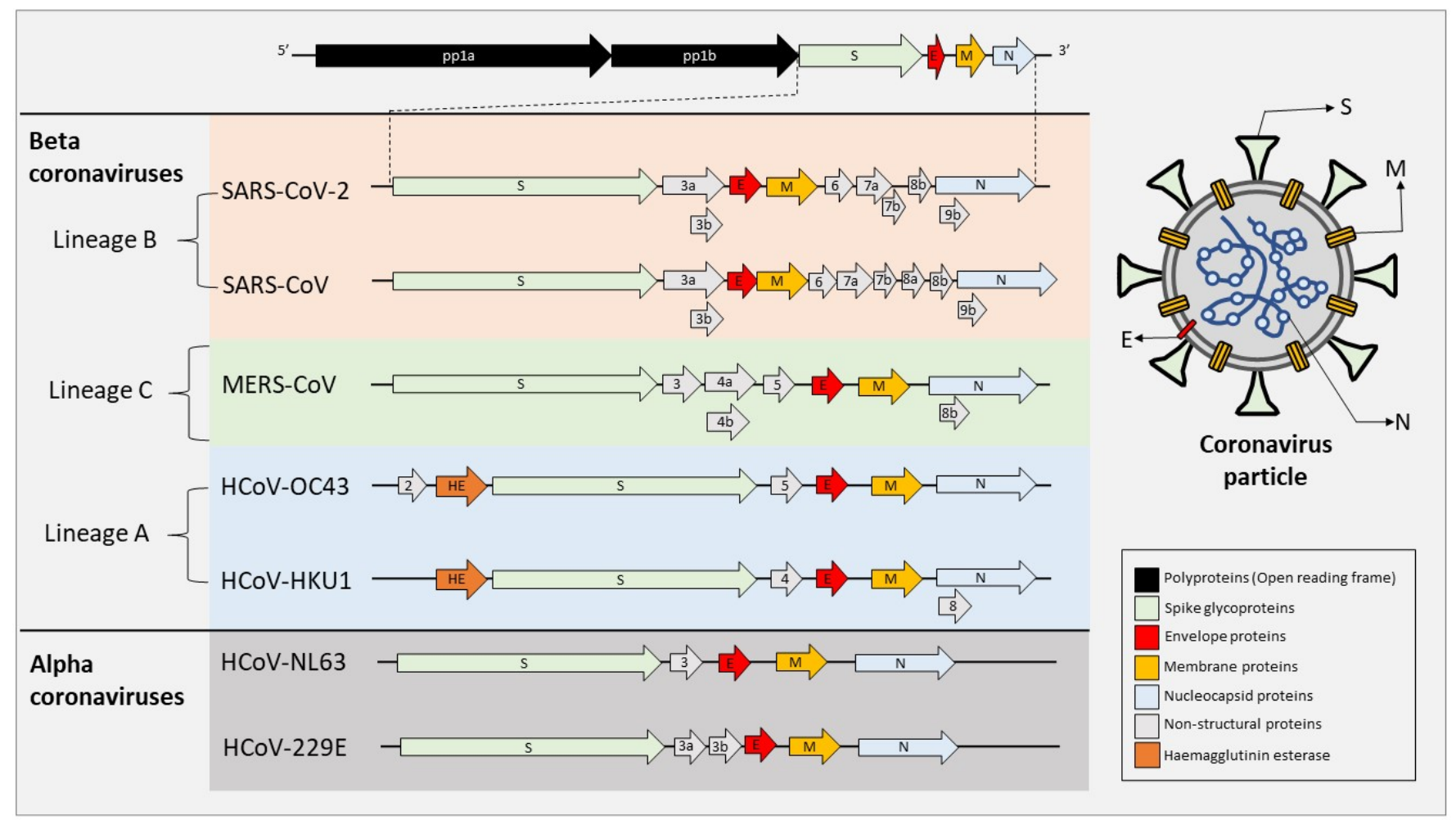

Figure 1: Schematic illustration of genome organization of the seven human coronaviruses (HCoVs) arranged in descending order from the most similar in gene sequence to SARS-CoV-2 above.(41) Coronaviruses genome is a single stranded, nonsegmented RNA genome, sized about 30 kilobases. This genome encodes structural proteins including: spike glycoprotein (S), envelope protein (E), membrane protein (M) , and nucleocapsid protein (N).(42) Also it encodes other accessory non-structural proteins that thought to have an immunomodulatory effects.(43)

At the genome level, SARS-CoV is $82.5 \%$ identical to SARS-CoV-2, while MERS-CoV, HCoV-OC43, HCoVHKU1, HCoV-NL63, and HCoV-229E are 69.6\%, 68.9\%, 67.6\%, 65.1\% and 65\% identical to SARS-CoV-2 respectively. Homology on proteomic level (structural and non-structural protein levels) is thought to be higher than genomic level. For example; most of SARS-CoV and SARS-CoV-2 proteins are considered highly homologues (95\%-100\%).(41) This could lead to high chances of sharing very similar immunogenic epitopes, which are recognized alike by immune cells and so induce pan-coronaviral immunity.(44)

Le Bert et al., 2020 have reported robust $C D 4^{+}$and $C D 8^{+}$memory $T$ cells against SARS-CoV that still contained in patients recovered from SARS 17 years ago, moreover these cells cross-reacted with Npeptides of SARS-CoV-2 in all patients.(45)

Rabets et al., 2021 have recently reported cross-reactive antibodies against MERS-CoV in convalescent plasma of COVID-19 patients.(44)

SARS-CoV-2 reactive CD4 ${ }^{+} T$ cells has been detected by Grifoni et al.,2020 in about $50 \%$ of unexposed 
individuals where their samples collected between 2015-2018, this cross-reactivity is suggested to be induced by the endemic HCoVs causing common cold.(39) Similar results have been reported by Weiskopf et al., 2020,(46) Braun et al., 2020,(47), Mateus et al.,2020(48) and Le Bert et al., 2020(45) but largerscale studies are still needed.

Besides cellular cross-reactions, SARS-CoV-2 spike(S2)-reactive IgG antibodies has been detected in different sera of SARS-CoV-2 unexposed individuals, also suggested to be due to previous infection with HCoVs. Interestingly, these IgG antibodies were more prevalent among children, which could explain their lower rates of infection.(49)

These cellular and humoral cross-reactions between SARS-CoV-2 and other human coronaviruses (HCoVs) support the hypothesis that SARS-CoV-2 can induce pan-coronaviral long lasting immunity (months-years) that could protect from other coronaviruses or alleviate their severity.

\section{Cross-immunity explains different COVID-19 presentations}

Cross-immunity is thought to be a reason for the variability of COVID-19 presentations among individuals and countries; in terms of disease duration, infectivity, severity and fatality.

Absence of infection although the exposure, along with asymptomatic and mild infections are thought to be due to different levels of pre-existing cross immunity.(50)

Age-wise, children are less susceptible to COVID-19. This observation could be explained not only by natural innate antibodies and maternal antibodies,(51) but also by the cross-protection induced by childhood vaccines like DPT(Diphtheria, Pertussis, Tetanus) or pneumococcal vaccines. $(52,53)$ This immunological cross-protection fades over time explaining the increase of vulnerability for COVID-19 with age. In addition, the four human endemic coronaviruses which have higher infection rates among young adults could explain COVID-19 age pattern as these viruses could induce pan-coronaviruses immunity .(49)

On population level, milder burden of COVID-19 within the Middle East is suggested to be due to crossreactivity with the regional endemic MERS-CoV.(54) High COVID-19 death rates among USA, UK, Italy and Spain and relatively lower rates among Africa, South America, China and Russia are hypothesised to be due to different BCG vaccination coverage.(55-57) The beneficial effect of BCG against COVID-19 is assumed to be due to the well reported non-specific trained immunity induced by the vaccine. However, these claims are based on observational studies with high risk of confounding bias. A hypothesis article by Root-Bernstein argued the role of BCG in explaining these geographical differences and suggest a role for pneumococcal vaccine instead.(53) For that, there are 20 ongoing clinical trials around the world looking for any impact of BCG vaccine on COVID-19. Interim analysis of one of these trials showed $53 \%$ reduction 
in SARS-CoV-2 infection rates in the BCG arm compared to placebo arm.(58)

Least developed countries in Africa and Asia are less than expected hit by COVID-19, this is possibly because they were naturally vaccinated through high exposure to endemic pathogens that cross-protect them from COVID-19 such as pneumococci and haemophilus.(59)

Interestingly, Pawlowski et al. have found that individuals recently vaccinated with non-COVID-19 vaccines (polio, Haemophilus Influinzae B, MMR, Varicella, PCV13, Geriatric Flu, or HepA-HepB) had lower SARS-CoV2 infection rates.(60) This is possible to occur due to cross-reactions of these vaccines with SARS-CoV-2.

\section{SARS-CoV-2 non-specific cross-reactions}

Flipping the aforementioned reports of cross-protection from COVID-19 induced by other pathogens and vaccines, it becomes more plausible to hypothesize that SARS-CoV-2 infection could naturally immunize immunocompetent individuals against other unrelated pathogens through the same mechanisms of epitopes sharing and/or innate immunity reprograming. Here are some shreds of promising evidence showing variable levels of similarity between different pathogens epitopes and predicted SARS-CoV-2 epitopes:

In silico prediction of potential COVID-19 cross-reactive epitopes based on proteomics mapping of variable unrelated pathogens (18 viruses and 7 bacteria) by Reche, 2020, 595 peptides from 8 viruses and 7 bacteria were found $\geq 80 \%$ identical with 551 peptides from SARS-CoV-2 in at least 8 residues (figure 2).

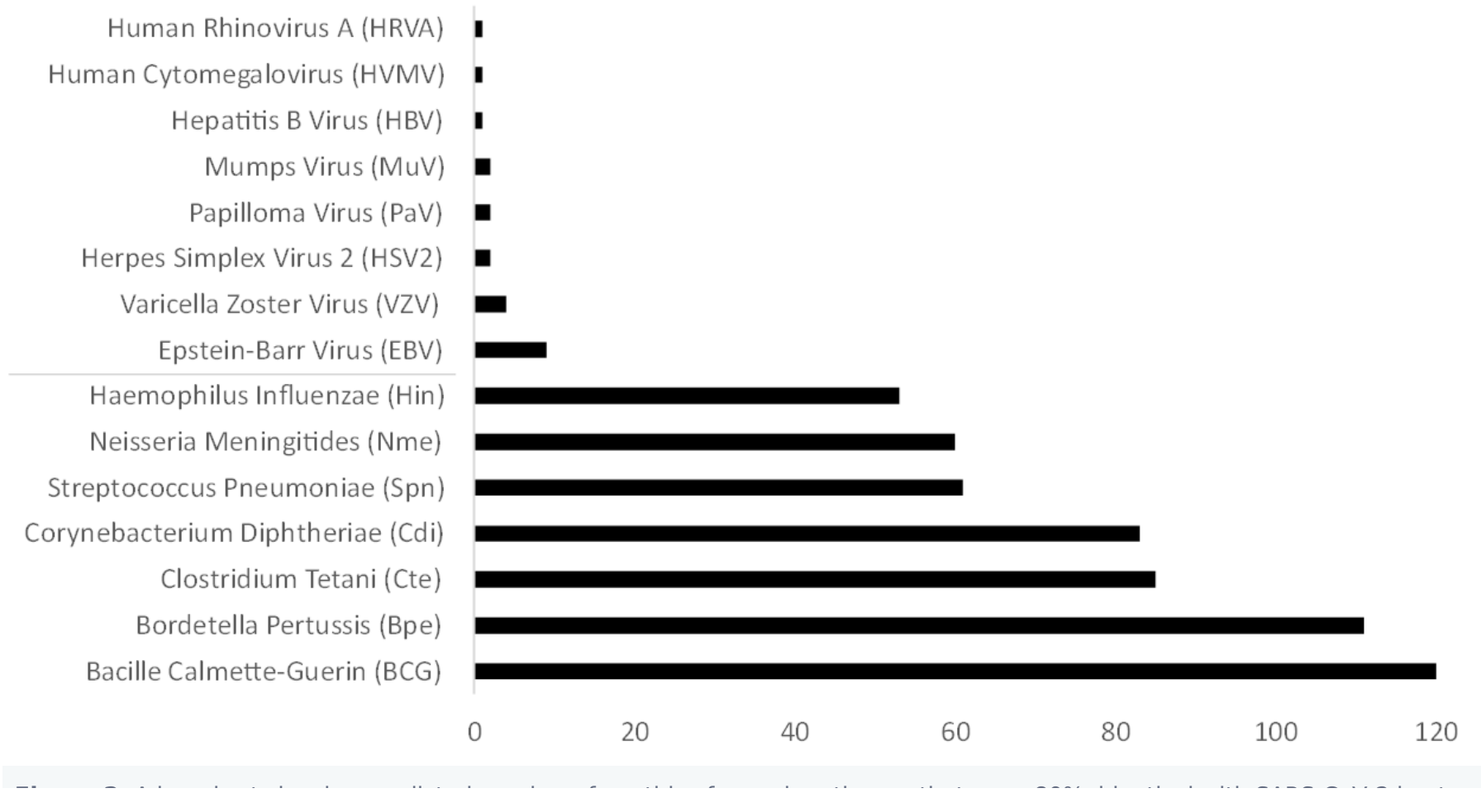


Figure 2: A bar chart showing predicted number of peptides for each pathogen that are $\geq 80 \%$ identical with SARS-CoV-2 in at least 8 residues. [Source of data: Reche, 2020].(52)

As noted in figure 2, bacteria -and similarly fungi and parasites- are expected to have higher levels of identical peptide sequences with SARS-CoV-2 compared to non-coronaviruses due to their larger proteome which increases the probability of matching and so the probability of cross-reactions. However, sharing peptides is one of many known and yet unknown determinants of cross-reactions. For example, in a study by Mateus et al., a peptide with $80 \%$ similarity with SARS-CoV-2 epitope failed to elicit any T-cell response while a peptide with $33 \%$ similarity succeeded.(48)

Successful cross-reaction requires processing of shared epitopes inside antigen presenting cells (APCs) and then presenting them in enough concentrations to the immune cells via human leukocyte antigen system (HLA). Considering this factor alone, peptides of large pathogens like fungi and bacteria have lower probability to be presented compared to viral peptides, as viruses are presented in excess to the immune system due to their intracellular multiplication pattern and speed.(61)

Stervbo et al. have used a computational approach comparing peptide sequences of the most common infectious pathogens in Europe (32 viruses, 26 bacteria, 11 fungi, and 2 parasites) to predicted HLA-1 and HLA-2 binding epitopes. Majority of these pathogens have shown different levels of matching. Top ten pathogens matching HLA-1 epitopes were: the four endemic HCoVs, Varicella-zoster virus (VZV), Rotavirus A, Influenza B, the fungi: Candida tropicalis and Cryptococcus neoformans, and the parasite Trichomonas vaginalis. Same pathogens ranked top ten for HLA-2 binding except for Epstein-Barr virus (EBV) replaced Trichomonas vaginalis. Then they compared epitopes of top ranked viruses to predicted SARS-CoV-2 epitopes, and all these viruses showed significant similarities at different edit distances.(61)

Similarly, in a study by Root-Bernstein, 2020, pneumococcal proteins (PspA, PspC, and PsaA) and a modified diphtheria toxin (CRM197) were found to have regions of significant similarity with SARS-CoV-2 spike protein (S), membrane protein (M) and replicase polyprotein 1a (1a). In the same study, sequence matches with SARS-CoV-2 were observed also in MMR, DPT and BCG vaccines.(62)Moreover, in a preprint small cohort study of 12 individuals by Sidhom et al., 2020, SARS-CoV-2-specific T-cell receptors (TCRs) have been detected to in-vitro cross-react with M1 peptides of Influenza virus.(63)

\section{Checkpoint}

Importantly, all of these should be taken as hypotheses, even if proven right, it does not call by any means for not following COVID-19 preventive measures on any level. It is only aimed to better understanding of the disease and our immune responses.

It is crucial to highlight that immune modulation and cross reactivity is not always beneficial. It is possible that suboptimal cross-reactive immune responses could weaken immunity for the favour of other 
pathogens or lead to less tolerance to self-antigens and induce autoimmune disease. For example, preexisting dengue antibodies were found to cross-react with zika virus and enhance its infectivity via antibody-dependent enhancement (ADE).(64) What defines the outcome of cross-reactivity is still poorly understood.

In COVID-19 context, suboptimal unfocused low avidity $\mathrm{CD}^{+}{ }^{+} \mathrm{T}$ cell responses suggested to be originated from previous infection with common cold coronaviruses, has shown to be linked to severe form of COVID19.(65) Moreover, co-infection with influenza A virus is reported to enhance the infectivity of SARS-CoV-2 in both cell culture and mice.(66) But fortunately, vaccination of recovered individuals is thought to optimize and boost immune responses to prevent such harmful outcomes.(37)

Further lab-based research is still needed to clarify determinants of cross-reactivity and cross-protection. Also Large scale cohort studies with matched controls are needed to elucidate the impact of COVID-19 on other diseases, and effect of pre-existing diseases on COVID-19 presentation.

\section{Anything else positive?}

Possible positive impact of COVID-19 is not limited to cross-immunity. Other hypotheses could be suggested among the scientific community for further testing. Some of them are on individual's health level like: " COVID-19 could promote healing in diseased organs (e.g. liver) through induction of stem cells activity", or on population level like:" The pattern of immune responses to COVID-19 along with presentation and severity of the disease could be used as rough measurement for stratification the population based on vulnerability for disease".

\section{Concluding remarks and future perspectives}

To conclude, it is highly possible that SARS-CoV-2 could lead to positive effects against other pathogens and diseases due to sharing of immunogenic epitopes or innate immunity reprogramming . The wide spread of SARS-CoV-2 and its variants around the world, along with the mass vaccination with variable forms of vaccines, besides other therapeutics and prolonged preventive measures, all could lead to new era of the evolution of the relationship between pathogen, disease and human immunity; in which new strains and pathogens could develop, compete or vanish. And on the other battle side, human immunity would get modulated and adapted to protect from certain pathogens and/or be more vulnerable to others. Some of these effects would be short-term and others could be transgenerational! To better understand and cope with that, further multilevel wide spread and prolonged research is highly needed in the upcoming years to define these changes in the worldwide disease map.

\section{References}

1. Dong E, Du H, Gardner L. An interactive web-based dashboard to track COVID-19 in real time. The 
Lancet Infectious Diseases. 2020;20(5):533-4.

2. Keeley AJ, Evans CM, De Silva TI. Asymptomatic SARS-CoV-2 infection: the tip or the iceberg? Thorax. 2020;75(8):621-2.

3. Tan L, Lin ZC, Ray J, Wesselingh R, Oxley TJ, McFadyen J, et al. Neurological implications of COVID19: a review of the science and clinical guidance. BMJ Neurology Open. 2020;2(2):e000101.

4. Mirza FN, Malik AA, Omer SB, Sethi A. Dermatologic manifestations of COVID-19: a comprehensive systematic review. International Journal of Dermatology. 2021;60(4):418-50.

5. Nalbandian A, Sehgal K, Gupta A, Madhavan MV, McGroder C, Stevens JS, et al. Post-acute COVID19 syndrome. Nature Medicine. 2021.

6. Nelson B. The positive effects of covid-19. BMJ. 2020:m1785.

7. Chiu N-C, Chi H, Tai Y-L, Peng C-C, Tseng C-Y, Chen C-C, et al. Impact of Wearing Masks, Hand Hygiene, and Social Distancing on Influenza, Enterovirus, and All-Cause Pneumonia During the Coronavirus Pandemic: Retrospective National Epidemiological Surveillance Study. Journal of Medical Internet Research. 2020;22(8):e21257.

8. Medzhitov R, Janeway C, Jr. Innate immune recognition: mechanisms and pathways. Immunological Reviews. 2000;173(1):89-97.

9. Tian Y, Grifoni A, Sette A, Weiskopf D. Human T Cell Response to Dengue Virus Infection. Frontiers in Immunology. 2019;10.

10. Frank SA. Immunology and Evolution of Infectious Disease.: Princeton (NJ): Princeton University Press; 2002.

11. Mihai, Quintin J, Jos. Trained Immunity: A Memory for Innate Host Defense. Cell Host \& Microbe. 2011;9(5):355-61.

12. Nankabirwa V, Tumwine JK, Mugaba PM, Tylleskär T, Sommerfelt H. Child survival and BCG vaccination: a community based prospective cohort study in Uganda. BMC Public Health. 2015;15(1):175. 13. Saron WAA, Rathore APS, Ting L, Ooi EE, Low J, Abraham SN, et al. Flavivirusserocomplex crossreactive immunity is protective by activating heterologous memory CD4 T cells. Science Advances. 2018;4(7):eaar4297.

14. Barton ES, White DW, Cathelyn JS, Brett-Mcclellan KA, Engle M, Diamond MS, et al. Herpesvirus latency confers symbiotic protection from bacterial infection. Nature. 2007;447(7142):326-9.

15. Uppuluri P, Lin L, Alqarihi A, Luo G, Youssef EG, Alkhazraji S, et al. The Hyr1 protein from the fungus Candida albicans is a cross kingdom immunotherapeutic target for Acinetobacter bacterial infection. PLOS Pathogens. 2018;14(5):e1007056.

16. Cornberg $\mathrm{M}$, Wedemeyer $\mathrm{H}$. Hepatitis $\mathrm{C}$ virus infection from the perspective of heterologous immunity. Current Opinion in Virology. 2016;16:41-8.

17. Wedemeyer H, Mizukoshi E, Davis AR, Bennink JR, Rehermann B. Cross-Reactivity between Hepatitis C Virus and Influenza A Virus Determinant-Specific Cytotoxic T Cells. Journal of Virology. 2001;75(23):11392-400. 
18. Singh S, Vedi S, Samrat SK, Li W, Kumar R, Agrawal B. Heterologous Immunity between Adenoviruses and Hepatitis C Virus: A New Paradigm in HCV Immunity and Vaccines. PLOS ONE. 2016;11(1):e0146404.

19. Clute SC. Cross-reactive influenza virus-specific CD8+ T cells contribute to lymphoproliferation in Epstein-Barr virus-associated infectious mononucleosis. Journal of Clinical Investigation. 2005;115(12):3602-12.

20. Benn CS, Netea MG, Selin LK, Aaby P. A small jab - a big effect: nonspecific immunomodulation by vaccines. Trends in Immunology. 2013;34(9):431-9.

21. Arts RJW, Moorlag SJCFM, Novakovic B, Li Y, Wang S-Y, Oosting M, et al. BCG Vaccination Protects against Experimental Viral Infection in Humans through the Induction of Cytokines Associated with Trained Immunity. Cell Host \& Microbe. 2018;23(1):89-100.e5.

22. Salem A, Nofal A, Hosny D. Treatment of Common and Plane Warts in Children with Topical Viable Bacillus Calmette-Guerin. Pediatric Dermatology. 2013;30(1):60-3.

23. Stensballe LG, Nante E, Jensen IP, Kofoed P-E, Poulsen A, Jensen H, et al. Acute lower respiratory tract infections and respiratory syncytial virus in infants in Guinea-Bissau: a beneficial effect of BCG vaccination for girls. Vaccine. 2005;23(10):1251-7.

24. Pittet LF, Curtis N. Does bacillus Calmette-Guérin vaccine prevent herpes simplex virus recurrences? A systematic review. Reviews in Medical Virology. 2021;31(1):1-9.

25. Berendsen ML, Van Gijzel SW, Smits J, De Mast Q, Aaby P, Benn CS, et al. BCG vaccination is associated with reduced malaria prevalence in children under the age of 5 years in sub-Saharan Africa. BMJ Global Health. 2019;4(6):e001862.

26. Kleinnijenhuis J, Quintin J, Preijers F, Joosten LAB, Jacobs C, Xavier RJ, et al. BCG-induced trained immunity in NK cells: Role for non-specific protection to infection. Clinical Immunology. 2014;155(2):213-9. 27. Kamat AM, Lerner SP, O’Donnell M, Georgieva MV, Yang M, Inman BA, et al. Evidence-based Assessment of Current and Emerging Bladder-sparing Therapies for Non-muscle-invasive Bladder Cancer After Bacillus Calmette-Guerin Therapy: A Systematic Review and Meta-analysis. European Urology Oncology. 2020;3(3):318-40.

28. Morra ME, Kien ND, Elmaraezy A, Abdelaziz OAM, Elsayed AL, Halhouli O, et al. Early vaccination protects against childhood leukemia: A systematic review and meta-analysis. Scientific Reports. 2017;7(1). 29. Villumsen M, Sørup S, Jess T, Ravn H, Relander T, Baker JL, et al. Risk of lymphoma and leukaemia after bacille Calmette-Guérin and smallpox vaccination: A Danish case-cohort study. Vaccine. 2009;27(49):6950-8.

30. Kremenovic M, Schenk M, Lee DJ. Clinical and molecular insights into BCG immunotherapy for melanoma. Journal of Internal Medicine. 2020;288(6):625-40.

31. El-Zein M, Parent ME, Benedetti A, Rousseau MC. Does BCG vaccination protect against the development of childhood asthma? A systematic review and meta-analysis of epidemiological studies. International Journal of Epidemiology. 2010;39(2):469-86. 
32. Faustman DL, Wang L, Okubo Y, Burger D, Ban L, Man G, et al. Proof-of-Concept, Randomized, Controlled Clinical Trial of Bacillus-Calmette-Guerin for Treatment of Long-Term Type 1 Diabetes. PLoS ONE. 2012;7(8):e41756.

33. Ristori G, Romano S, Cannoni S, Visconti A, Tinelli E, Mendozzi L, et al. Effects of Bacille CalmetteGuerin after the first demyelinating event in the CNS. Neurology. 2014;82(1):41-8.

34. Ni L, Ye F, Cheng M-L, Feng Y, Deng Y-Q, Zhao H, et al. Detection of SARS-CoV-2-Specific Humoral and Cellular Immunity in COVID-19 Convalescent Individuals. Immunity. 2020;52(6):971-7.e3.

35. Rydyznski Moderbacher C, Ramirez SI, Dan JM, Grifoni A, Hastie KM, Weiskopf D, et al. AntigenSpecific Adaptive Immunity to SARS-CoV-2 in Acute COVID-19 and Associations with Age and Disease Severity. Cell. 2020;183(4):996-1012.e19.

36. Dan JM, Mateus J, Kato Y, Hastie KM, Yu ED, Faliti CE, et al. Immunological memory to SARS-CoV-2 assessed for up to 8 months after infection. Science. 2021;371(6529):eabf4063.

37. Stamatatos L, Czartoski J, Wan Y-H, Homad LJ, Rubin V, Glantz H, et al. mRNA vaccination boosts cross-variant neutralizing antibodies elicited by SARS-CoV-2 infection. Science. 2021:eabg9175.

38. Babiker A, Marvil CE, Waggoner JJ, Collins MH, Piantadosi A. The Importance and Challenges of Identifying SARS-CoV-2 Reinfections. Journal of Clinical Microbiology. 2020;59(4).

39. Grifoni A, Weiskopf D, Ramirez SI, Mateus J, Dan JM, Moderbacher CR, et al. Targets of T Cell Responses to SARS-CoV-2 Coronavirus in Humans with COVID-19 Disease and Unexposed Individuals. Cell. 2020;181(7):1489-501.e15.

40. Grifoni A, Sidney J, Zhang Y, Scheuermann RH, Peters B, Sette A. A Sequence Homology and Bioinformatic Approach Can Predict Candidate Targets for Immune Responses to SARS-CoV-2. Cell Host \& Microbe. 2020;27(4):671-80.e2.

41. Kaur N, Singh R, Dar Z, Bijarnia RK, Dhingra N, Kaur T. Genetic comparison among various coronavirus strains for the identification of potential vaccine targets of SARS-CoV2. Infection, Genetics and Evolution. 2021;89:104490.

42. Weiss SR, Leibowitz JL. Chapter 4 - Coronavirus Pathogenesis. In: Maramorosch K, Shatkin AJ, Murphy FA, editors. Advances in Virus Research. 81: Academic Press; 2011. p. 85-164.

43. Liu DX, Fung TS, Chong KK-L, Shukla A, Hilgenfeld R. Accessory proteins of SARS-CoV and other coronaviruses. Antiviral Research. 2014;109:97-109.

44. Rabets A, Bila G, Grytsko R, Samborskyy M, Rebets Y, Vari SG, et al. The Potential of Developing Pan-Coronaviral Antibodies to Spike Peptides in Convalescent COVID-19 Patients. Archivum Immunologiae et Therapiae Experimentalis. 2021;69(1).

45. Le Bert N, Tan AT, Kunasegaran K, Tham CYL, Hafezi M, Chia A, et al. SARS-CoV-2-specific T cell immunity in cases of COVID-19 and SARS, and uninfected controls. Nature. 2020;584(7821):457-62.

46. Weiskopf D, Schmitz KS, Raadsen MP, Grifoni A, Okba NMA, Endeman H, et al. Phenotype and kinetics of SARS-CoV-2-specific T cells in COVID-19 patients with acute respiratory distress syndrome. Sci Immunol. 2020;5(48):eabd2071. 
47. Braun J, Loyal L, Frentsch M, Wendisch D, Georg P, Kurth F, et al. Presence of SARS-CoV-2-reactive T cells in COVID-19 patients and healthy donors. 2020.

48. Mateus J, Grifoni A, Tarke A, Sidney J, Ramirez SI, Dan JM, et al. Selective and cross-reactive SARSCoV-2 T cell epitopes in unexposed humans. Science. 2020;370(6512):89-94.

49. Ng KW, Faulkner N, Cornish GH, Rosa A, Harvey R, Hussain S, et al. Preexisting and de novo humoral immunity to SARS-CoV-2 in humans. Science. 2020;370(6522):1339-43.

50. Lipsitch M, Grad YH, Sette A, Crotty S. Cross-reactive memory T cells and herd immunity to SARSCoV-2. Nature Reviews Immunology. 2020;20(11):709-13.

51. Carsetti R, Quintarelli C, Quinti I, Piano Mortari E, Zumla A, Ippolito G, et al. The immune system of children: the key to understanding SARS-CoV-2 susceptibility? The Lancet Child \& Adolescent Health. 2020;4(6):414-6.

52. Reche PA. Potential Cross-Reactive Immunity to SARS-CoV-2 From Common Human Pathogens and Vaccines. Frontiers in Immunology. 2020;11.

53. Root-Bernstein R. Age and Location in Severity of COVID-19 Pathology: Do Lactoferrin and Pneumococcal Vaccination Explain Low Infant Mortality and Regional Differences? BioEssays. 2020;42(11):2000076.

54. Barry M, Ebrahim SH, Almohaya A, Alhijji A, Akkielah L, Alrajhi A, et al. Clinical Characteristics and Outcome of Hospitalized COVID-19 Patients in a MERSCoV Endemic Area. Journal of Epidemiology and Global Health. 2020.

55. Islam MZ, Zahan MKE, AI-Bari MAA. Convergence between global BCG vaccination and COVID-19 pandemic. Journal of Medical Virology. 2021;93(3):1496-505.

56. Escobar LE, Molina-Cruz A, Barillas-Mury C. BCG vaccine protection from severe coronavirus disease 2019 (COVID-19). Proceedings of the National Academy of Sciences. 2020;117(30):17720-6. 57. Kumar A, Misra S, Verma V, Vishwakarma RK, Kamal VK, Nath M, et al. Global impact of environmental temperature and BCG vaccination coverage on the transmissibility and fatality rate of COVID-19. PLOS ONE. 2020;15(10):e0240710.

58. Sepsis HIftSo. Bacillus Calmette-guérin Vaccination to Prevent COVID-19 (ACTIVATEII) (NCT04414267) 2021 [Available from: https://clinicaltrials.gov/ct2/show/NCT04414267.

59. Root-Bernstein R. Pneumococcal and Influenza Vaccination Rates and Pneumococcal Invasive Disease Rates Set Geographical and Ethnic Population Susceptibility to Serious COVID-19 Cases and Deaths. Vaccines. 2021;9(5):474.

60. Pawlowski C, Puranik A, Bandi H, Venkatakrishnan AJ, Agarwal V, Kennedy R, et al. Exploratory analysis of immunization records highlights decreased SARS-CoV-2 rates in individuals with recent nonCOVID-19 vaccinations. Scientific Reports. 2021;11(1).

61. Stervbo U, Rahmann S, Roch T, Westhoff TH, Babel N. Epitope similarity cannot explain the preformed T cell immunity towards structural SARS-CoV-2 proteins. Scientific Reports. 2020;10(1).

62. Root-Bernstein R. Possible Cross-Reactivity between SARS-CoV-2 Proteins, CRM197 and Proteins in 
Pneumococcal Vaccines May Protect Against Symptomatic SARS-CoV-2 Disease and Death. Vaccines. 2020;8(4):559.

63. Sidhom J-W, Baras AS. Analysis of SARS-CoV-2 specific T-cell receptors in ImmuneCode reveals cross-reactivity to immunodominant Influenza M1 epitope. 2020.

64. Priyamvada L, Quicke KM, Hudson WH, Onlamoon N, Sewatanon J, Edupuganti S, et al. Human antibody responses after dengue virus infection are highly cross-reactive to Zika virus. Proceedings of the National Academy of Sciences. 2016;113(28):7852-7.

65. Bacher P, Rosati E, Esser D, Martini GR, Saggau C, Schiminsky E, et al. Low-Avidity CD4+ T Cell Responses to SARS-CoV-2 in Unexposed Individuals and Humans with Severe COVID-19. Immunity. 2020;53(6):1258-71.e5.

66. Bai L, Zhao Y, Dong J, Liang S, Guo M, Liu X, et al. Coinfection with influenza A virus enhances SARS-CoV-2 infectivity. Cell Research. 2021;31(4):395-403. 